\title{
Reference intervals of hematological variables for healthy antepartum and postpartum women
}

\begin{abstract}
Objective: To report the reference intervals for antepartum and postpartum hematological variables.

Material and methods: The series consists of 488 women with a normal pregnancy duration of $\geqq 37$ weeks and a normal delivery with a newborn weight $>2500 \mathrm{~g}$. Blood samples were obtained 24 hours before birth and 24 hours after birth. The following indicators were analyzed: white blood cell (WBC), red blood cell (RBC), hemoglobin (HB), hematocrit (HCT), platelet (PLT), and neutrophil (NE) counts. Reference values were calculated as the mean $\pm 1.96 \times \mathrm{SD}$.

Results: Compared with antepartum measurements, RBC, Hb, PLT, and HCT values declined at 24 hours postpartum $(\mathrm{P}<0.05)$, whereas $\mathrm{WBC}$ and NE values significantly increased $(\mathrm{P}<0.05)$.

Conclusion: The characteristic changes occurring in hematological indices at $24 \mathrm{~h}$ antepartum and $24 \mathrm{~h}$ postpartum are described in this study. The results may be used as reference values in the assessment of blood infection in antepartum and postpartum women.
\end{abstract}

Keywords: normal pregnancy women, hematological variables, reference intervals
Volume I Issue 3 - 2014

Qingchun Deng, Li Deng, Cheng Chen, Shan Meng, Yinglian Pan, Qing Chang

Department of Gynecology and Obstetrics, Third Military Medical University, China

Correspondence: Chang Qing, Department of Obstetrics and Gynecology, Southwest Hospital, Third Military Medical University, Chongqing, 400038, China, Tel +86-23-68765399, Fax +86-23-68765399, Email cqli99@yahoo.com.cn

Received: October 19,2014 | Published: December 04, 2014

\section{Introduction}

Hematological variables change significantly as a pregnancy progresses. Similarly, hematological variables also change significantly $24 \mathrm{~h}$ after birth. These changes are partly attributed to blood dilution, nutrition intake during the periods of pregnancy, and bleeding in the process of delivery. ${ }^{1,2} \mathrm{~A}$ thorough understanding of the hematological changes can serve as a guide for disease diagnosis and the rational use of drug postpartum. To date, the reference intervals for antepartum and postpartum hematological variables have not been fully investigated. Here, we describe the hematological variables $24 \mathrm{~h}$ before birth and after birth in this study and then analyze the reference intervals for hematological variables to provide favorable standards for antepartum and postpartum disease diagnosis.

\section{Materials and methods}

For this study, 488 women patients were randomly selected from the Department of Gynecology at the Southwest Hospital from August 2011 to December 2012. All participants fulfilled following inclusion criteria: healthy, 21 to 35 years old, weighs between 48 to 76kilograms, experiencing a normal pregnancy, spontaneous delivery, no postpartum hemorrhage determined, no antibiotic use 2 weeks before delivery, normal temperature before labor, no other diseases, and no serious pregnancy complications and labor complication. Every woman selected for this study provided informed voluntarily consent and completed the questionnaires.

All selected women completed routine blood work $24 \mathrm{~h}$ before birth and $24 \mathrm{~h}$ after birth. We analyzed hematological variables, including WBC, RBC, HB, PLT, NE, and HCT, and explored reference intervals. Blood samples were collected in a routine method. Venipuncture was performed in an antecubital vein. The blood samples were collected in
BD purple cap vacuum tubes (2ml, EDTA-k2 anticoagulation). Blood samples were analyzed by an automated cell counter (Sysmex XE2100, Japan) and reagents (Sysmex, Japan).

\section{Statistics}

Statistical analysis was performed using SPSS 13.0 statistical software (SPSS, Chicago, IL, USA). For single samples, data were assessed using the Kolmogorov-Smirnov test. The normal distributions of the hematological values were evaluated by histograms. Reference intervals were calculated as the mean $\pm 1.96 \times \mathrm{SD} .{ }^{3}$ Paired antepartum and postpartum hematological variables were assessed using a paired t-test.

\section{Results}

\section{Clinical characteristics}

A total of 488 patients met the inclusion criteria. All patients experienced a full-term pregnancy and vaginal delivery. In total, $168(34.4 \%)$ patients were administered epidural analgesia, and 189 (38.7\%) women experienced an episiotomy. The mean volume of postpartum hemorrhage in two hours was $215.4 \mathrm{ml}$, and postpartum hemorrhage was not noted in any patient. In total, 39 (7.8\%) patients experienced a fever greater than $37.3^{\circ} \mathrm{C}$. The clinical characteristics of all women are presented in Table 1.

\section{The reference intervals of hematological variables}

We established normal reference intervals for antepartum and postpartum hematological variables in healthy women (Table 2 ). The WBC, RBC, HB, PLT, NE, and HCT reference intervals in non-pregnant women are $4 \times 109 \sim 10 \times 109 / \mathrm{L}, 3.5 \times 1012 \sim 5.0 \times 1012 / \mathrm{L}, 110 \sim 150 \mathrm{~g} / \mathrm{L}$, $100 \sim 300 \times 109 / \mathrm{L}, \quad 50 \sim 70 \%$, and $37 \sim 48 \%$, respectively. However, 
our antepartum reference interval results for WBC, RBC, HB, PLT, NE, and HCT are $3.42 \times 109 \sim 15.69 \times 109 / \mathrm{L}, 3.168 .93 \sim 251.77 \times 109 / \mathrm{L}$, 93.86 146.25 $\mathrm{g} / \mathrm{L}, \quad 59.8 \sim 286.2 \times 109 / \mathrm{L}, \quad 4.84 \sim 18.14 \times 109 / \mathrm{L}$ $(70.81 \sim 90.73 \%)$, and $24.56 \sim 47 \sim 4.61 \times 1012 / \mathrm{L}$, respectively. These respective postpartum values are $7.06 \sim 21.16 \times 109 / \mathrm{L}$,

Table I Characteristics of all vaginal deliveries during the study period
$2.72 \sim 4.39 \times 1012 / \mathrm{L}, \quad 79.49 \sim 137.89 \mathrm{~g} / \mathrm{L}, \quad 0.81 \%, \quad 4.84 \sim 18.14 \times 109 / \mathrm{L}$ (70.81 90.73\%), and 24.56 47 4.61×1012/L. These lower and upper reference limits significantly differ among non-pregnant, antepartum, and postpartum women.

\begin{tabular}{ll}
\hline Characteristic & $\mathbf{N}=\mathbf{4 8 8}$, value $^{*}$ \\
\hline Maternal age (years) & $26.2 \pm 4.8$ \\
Maternal BMl $\left(\mathrm{kg} / \mathrm{m}^{2}\right)$ & $31.6 \pm 5.8$ \\
Gestational age (days) & $272.3 \pm 10.5$ \\
Epidural & $168(34.4 \%)$ \\
Episiotomy & $189(38.7 \%)$ \\
Volume of postpartum hemorrhage in two hours $(\mathrm{ml})$ & $215.4(42.6)$ \\
T>37.3 ${ }^{\circ} \mathrm{C}$ & $39(7.8 \%)$ \\
CRP & $46(9.4 \%)$ \\
Birth weight (grams) & $3716.5 \pm 439.2$ \\
Birth height $(\mathrm{cm})$ & $49.8 \pm 1.3$ \\
Apgar scores & $9.4 \pm 0.3$ \\
\hline
\end{tabular}

BMI, body mass index; CRP, C-response protein

$*$ Mean \pm SD or $\mathrm{N}(\%)$

Table 2 Mean and 95\% confidence intervals for hematological variables in healthy antepartum and postpartum women

\begin{tabular}{|c|c|c|c|c|c|}
\hline \multirow{2}{*}{ Parameters } & \multicolumn{2}{|c|}{$37-40$ weeks $(n=488)$} & \multicolumn{2}{|c|}{$24 \mathrm{~h}$ after postpartum $(n=488)$} & \multirow{2}{*}{ P-value } \\
\hline & $\overline{\mathbf{x}} \pm \mathbf{s}$ & $95 \% \mathrm{Cl}^{*}$ & $\overline{\mathbf{x}} \pm \mathbf{s}$ & $95 \% \mathrm{Cl}$ & \\
\hline WBC (×109/L) & $9.56 \pm 3.07$ & $3.42 \sim 15.69$ & $|4.1| \pm 3.53$ & $7.06 \sim 21.16$ & $<0.01$ \\
\hline $\mathrm{RBC}(\times 1012 / \mathrm{L})$ & $3.89 \pm 0.36$ & $3.17 \sim 4.61$ & $3.56 \pm 0.42$ & $2.72 \sim 4.39$ & $<0.05$ \\
\hline $\mathrm{HB}(\mathrm{g} / \mathrm{L})$ & $120.05 \pm 13.10$ & $93.86 \sim 146.25$ & $108.69 \pm 14.60$ & $79.49 \sim 137.89$ & $<0.01$ \\
\hline $\operatorname{PLT}(\times 1012 / L)$ & $|73.0| \pm 56.60$ & $59.8 \sim 286.2$ & $|60.35 \pm 45.7|$ & $68.93 \sim 251.77$ & $<0.01$ \\
\hline NE (\%) & $75.88 \pm 8.26$ & $59.35 \sim 92.4 \mathrm{I}$ & $80.77 \pm 4.98$ & $70.81 \sim 90.73$ & $<0.01$ \\
\hline NE\# (×109/L) & $7.43 \pm 2.94$ & $1.56 \sim 13.30$ & $11.49 \pm 3.33$ & $4.84 \sim 18.14$ & $<0.01$ \\
\hline HCT (\%) & $35.89 \pm 3.67$ & $28.55 \sim 43.23$ & $32.68 \pm 4.06$ & $24.56 \sim 40.81$ & $<0.05$ \\
\hline
\end{tabular}

$* 95 \% \mathrm{Cl}=\overline{\mathrm{x}} \pm 1.96 \mathrm{~s}$

\section{Discussion}

Anemia is the most common hematological problem in the process of pregnancy. From this study, we also found changes of the hematological variables for antepartum and postpartum women. Therefore, for antepartum and postpartum women, there will be a mistake to classify normal and abnormal according to no-pregnant women' reference values. ${ }^{4,5}$ Better understanding the reference interval of hematological variables is essential for disease diagnosing and treatment in antepartum and postpartum women.

Here, we demonstrated that physiologic anemia is typically observed in normal antepartum and postpartum women due to blood dilution. In fact, this outcome does not seriously affect pregnancy outcome. ${ }^{6}$ During the period of a normal pregnancy, plasma volumes red blood cells gradually increase. Physiologic anemia occurs when the plasma volume increases more rapidly than the increase in red blood cells occurs. ${ }^{7,8}$ Intervention behaviors increased during the process of delivery along with advancing perinatology. For example, the administrations of prophylactic antibiotics to women who undergo vaginal delivery not only enhance economic burden but also influence postpartum breast feeding. In a normal pregnancy, our results indicate that white cell counts increase in antepartum and postpartum women. The mechanism of increased white cell counts has not been clearly investigated. A complicated physiological procedure results in increased white cell counts during the gestational period. A series of changes occurs in the genital system, endocrine system, and metabolic processes during gestation. Therefore, white cells obviously increase because white cells accept a series of stimulatory signals as gestation 
progresses. ${ }^{9}$ The previous studies indicate that the increase in white cells during gestation is associated with the release of leukocytes from marginal pools. ${ }^{10}$ Thus, it is unreasonable to use antibiotics to prevent infection during birth. Our data indicate that the white cell counts were more significantly increased in postpartum women compared with antepartum women, whereas CRP and temperature generally remained at normal levels. These changes are possibly attributed to physiological fluctuations and reflect enhanced immune functions.

In conclusion, we presented updated reference intervals for various hematological variables in healthy pregnant women at 24 hours before and after birth. Our results potentially provide novel reference levels for the diagnosis and treatment of diseases in antepartum and postpartum women.

\section{Acknowledgments}

None.

\section{Conflicts of interest}

The authors declare there is no conflict of interests.

\section{References}

1. Robeck TR, Nollens HH. Hematological and serum biochemical analytes reflect physiological challenges during gestation and lactation in killer whales (orcinus orca). Zoo Biol. 2013;32(5):497-509.
2. Sharma SK, Philip J, Wiley J. Thromboelastographic changes in healthy parturients and postpartum women. Anesth Analg. 1997;85(1):94-98.

3. Katayev A. Estimating reference intervals. Am $J$ Clin Pathol. 2010;134(2):351

4. Townsley DM. Hematologic complications of pregnancy. Semin Hematol. 2013;50(3):222-231.

5. Chang YH, Yang SH, Wang TF, et al. Complete blood count reference values of cord blood in Taiwan and the influence of gender and delivery route on them. Pediatr Neonatol. 2011;52(3):155-160.

6. Shields RC, Caric V, Hair M, et al. Pregnancy-specific reference ranges for haematological variables in a Scottish population. J Obstet Gynaecol. 2011;31(4):286-289

7. Scholl TO, Reilly T. Anemia, iron and pregnancy outcome. $J$ Nutr. 2000;130(2S Suppl):443S-447S.

8. Milman N, Bergholt T, Byg KE, et al. Reference intervals for haematological variables during normal pregnancy and postpartum in 434 healthy danish women. Eur J Haematol. 2007;79(1):39-46.

9. Solves P, Lopez M, Mirabet V, et al. Characteristics of umbilical cord blood units collected from preterm deliveries. Gynecol Obstet Invest. 2009;68(3):181-185.

10. Marin GH, Fazio P, Rubbo S, et al. Prevalence of anaemia in pregnancy and analysis of the underlying factors. Aten Primaria. 2002;29(3):158-163. 